\title{
Analysis on the Evolution of Urban Land Structure and Economic Driving Force in Changde
}

\author{
Jun XIAO \\ College of Economics and Management, Sichuan Agricultural University \\ Ya'an 625014, China \\ Tel: 86-159-5189-2959 E-mail: Dina_bestfriend@yahoo.com.cn \\ JIAN-Qiang Li (Corresponding author) \\ College of Economics and Management, Sichuan Agricultural University \\ Ya'an 625014, China \\ Tel: 86-139-8161-6343Ｅ-mail: ljq9801@163.com
}

JIANG-Long CHEN

Nanjing Institute of Geography \& Limnology, Chinese Academy of Sciences, Nanjing 210008, Jiangsu, China

Tel: 86-139-1298-1319 E-mail: jlchen@niglas.ac.cn

Peng TANG

College of Economics and Management, Sichuan Agricultural University

Ya'an 625014, China

Tel: 86-138-8244-6674Ｅ-mail: tangpeng19861009@hotmail.com

The research is financed by the National Natural Science Foundation Project of the People's Republic of China. No. 70873120

\begin{abstract}
Based on information entropy theory, the dynamically evolutional characteristics of urban land structure were analyzed in the time and space from 2001-2008, in Changde city. In time, the entropy and equilibrium of urban land information increased first and then decreased, with a slight fluctuation in 2007. The overall entropy and equilibrium showed a relatively stable trend, but the degree of dominance and equilibrium were the opposite trend. In space, information entropy, degree of equilibrium and dominance showed significant differences in each designated town of Changde. Further by constructing a multiple regression model to investigate economic driving force of the urban land evolution in Changde city, this paper found that the level of urbanization was the most important economic driver, followed by investment in fixed assets and GDP.
\end{abstract}

Keywords: Changde city, Urban land, The evolution of structure, Economic driving force

\section{Introduction}

Land use structure is the sum of the proportion of area of national economic sectors and their mutual relationship, which is the composition of the various sites according to certain methods of collection (Wan-mao, Han Tongkui, 2002). Urban land use structure not only for the relationship between space and form different combinations of patterns, but also of the time evolution and processes. In its formation and development process, urban land use structure subject to a variety of geographic processes and human activities (Zhang study, Yao Hui, 2006, pp.74-78). As the land use in the region showed clearly on the spatial and temporal variation Characteristics (Wang Haihong, Yan-ni 2008, pp.1-6), in order to do more reasonable and effective use of land resources, configuration, and scholars of different land types of structures such as the evolution and influence factors were quantified research. These studies mainly adopt information entropy, factor analysis, multiple factors such as gray correlation analysis method, on the various sites on the evolution of time series analysis. Analysis of driving 
forces, the Cuiyou Jun (2009, pp.132-152) used multivariate nonparametric local linear estimation method (Cui Youjun, 2009, pp.132-152), and more scholars used a multiple regression analysis drivers (Wang Qun, Wan-mao, 2005, pp.21-25), but still only used time series data.

Changde City, Hunan Province locates northwest of China, Dongting Lake and the west, the terrain dominated by plains. All under the jurisdiction are Wuling District, Ding District, Tsu, security village, Hanshou, Li County, Linli, Taoyuan, Shek Mun 9 districts and counties (cities). In 2008, the total area is 1,818,982.48 hectares of land, of which, construction land 168411.12 hectares, accounting for $9.26 \%$ of the total land area, urban land 18051.49 hectares, accounting for the city construction land area of $10.72 \%$, accounting for $0.60 \%$ of total land area. To achieve the city's GDP 104,969,750,000 yuan, 21.48 percent over the previous year. As the economic strength of Changde City, the significant increase in fixed assets investment increased. The scale of urban land will present a growing trend. The face of increasing demands for urban land. How can the structure of urban land in Changde City, more rational, efficient, and orderly be very important and urgent issue.

This article is based on the background, the use of Changde City, the City and the towns of urban land for each year of data, using information entropy method to calculate the 2001-2008 structure of urban land in Changde City, the information entropy, and equilibrium degree of dominance. And on this basis, the establishment of multiple linear regression model to study urban land in Changde City, the economic drivers of evolution. To better and more comprehensive grasp of Changde City, the temporal and spatial evolution of urban land use characteristics, reveals the evolution of urban land use driven by various economic factors effect on the rational optimization of urban land readjustment in Changde City. The structure has some inspiration.

\section{Research methods and data sources}

\subsection{Research Methods}

\subsubsection{Information Entropy}

The extent of land use systems can be ordered to describe and depict the information entropy, usually referred to as Shannon entropy defined by information entropy. Created based on Shannon information theory, information entropy is a measure of uncertainty. The greater the uncertainty the more disordered, so the information entropy is a measure of disorder. The system must have a direction to the orderly flow of negative entropy input (wetland restoration, Wu-fang, 2003, pp.112-117). Set up a total area of urban land area for the $\mathrm{S}$ to the region is divided into $n$ kinds of urban land use types, various types of urban land use area of $\operatorname{Si}(i=1,2, \ldots, n)$, then:

$$
\sum_{i=1}^{n} S_{i}=S
$$

Various types of urban land area of the region and the proportion of the total area of urban land use $\mathrm{Pi}$ is as follows:

$$
P_{i}=\frac{S_{i}}{S}=\frac{S_{i}}{\sum_{i=1}^{n} S_{i}}
$$

Clearly, $\sum_{i=1}^{n} P_{i}=1$, the average amount of information known as the Shannon entropy, information entropy of the expression is:

$$
H=-\sum_{i=1}^{n} P_{i} \ln P_{i}
$$

The formula: $\mathrm{H}$ for the information entropy, in units of bits, the size reflects the amount of urban land use types and the types of urban land area, its distribution. When the town land area equal to that $\mathrm{S} 1=\mathrm{S} 2=\ldots=\mathrm{Sn}=\mathrm{S} / \mathrm{n}$, the maximum entropy, that is $H_{\max }=\ln n$. Thus, the more types of urban land, the greater the entropy. The lower the information entropy value is, the higher degree of regional land use system will be, it is good for human life and production (Bo Lu, Jin Yong Zhang, 2008, pp.19-21).

\subsubsection{Balance Degree and dominance}

Entropy reflects the complexity of urban use, If the land classification or land classification of different land use types in the same amount, but not at the same time, the lack of comparability of information entropy (Maoliang 
Xiang, Lin Yanhua, 2008, pp.48-51), and balanced and dominant of the description of the urban land area the size of the differences between types, as well as the functions of the type of structure pattern, based on information entropy. Therefore, the balanced degree and dominance were introduction (Li Jiang, Qing-Sheng Guo, 2002, pp.393-397).

$$
J=H / H_{m}=-\left[\sum_{i=1}^{n} P_{i} \ln P_{i}\right] / \ln n
$$

The formula: $\mathrm{J}$ is balancing, which is the actual entropy and maximum entropy ratio. That is the equilibrium level of urban land use. Because $H \leq H_{m}$, we can see $0 \leq J \leq 1$. When $\mathrm{J}=0$, the land is the most unequal state, $\mathrm{J}=1$, then the type of urban land to achieve the desired balance. Accordingly, the dominance of the expression is:

$$
I=1-J
$$

I reflected dominance within the region of one or several dominant land use type of land the extent of the region, and a balanced degree of opposite meaning.

\subsection{Urban land classification and data sources}

According to "Statistical Yearbook of Changde" Changde will land into residential land types, public facilities, land, industrial land, warehouse space, external transport sites, roads and squares land, municipal facilities, green space and urban land types 8,2001 - 2008, the City and the towns of Changde, the land type data were derived from the "Statistical Yearbook of Changde City" (2002-2009).

\section{Changde City, the dynamic evolution of urban land use structure of Temporal and Spatial}

\subsection{Differentiation of timing}

According to the formula (1) - (5), nearly 8 years of Changde City, the structure of urban land use information entropy, and equilibrium degree of dominance were calculated (Table 1).Changde City, 2001-2008 by the urban land use structure and its information entropy, equilibrium degree of change with the dominance analysis (Table 1, Figure 1), can be seen in Changde City, the information entropy and equilibrium degree of both experienced decreases later change, and reached a maximum in 2005. Among them, 2001, the lowest entropy and equilibrium, $\mathrm{H}$ is $1.8010, \mathrm{~J}$ to 0.8661 to the maximum value of information entropy in 2005 , reaching 1.8427 and 0.8862 , followed by a downward trend. Accordingly, dominance decreased and then increased the trend; in 2001 the largest .1339 to 2005 reached the minimum, as 0.1138 .

Specifically, Changde City in 2001, the structure of urban land use information entropy and the lowest balance, indicated that the structure of Changde city land a higher degree of order. But the land type of imbalance, poor balance, accounted for superiority; Since then, the structure of urban land use information entropy and equilibrium degree increased gradually, indicating the type of urban land use change in large, and continuously adjust the proportion of various types of land; Structure of urban land use information entropy and equilibrium degree to reach the highest point in 2005, which indicated the greatest changes in urban land use types, urban land use structure of the least orderly, balanced utilization of urban land the highest; 2005-2006, due to public facilities, land and industrial land increased significantly increased by $1.76 \%$ and $4.16 \%$, and the sharp decline in residential land was $2.79 \%$, resulting in the structure of urban land in 2006 a sharp decline in entropy, equilibrium is started decline, dominance increased, reflecting the region of one or several dominant land use type of level land in the region increased,By 2008, residential land on the increase again over the major changes in industrial sites, public facilities and roads and squares have also been increased, leading to entropy rise slowly and then decreased, the structure of urban land increased degree of order.

\subsection{Spatial Characteristics}

From Table 2, Table 3 to analyze the city and towns of the urban land use structure information entropy and equilibrium degree of spatial differentiation: In space, the structure of urban land use information entropy and equilibrium degree of movement is consistent, balanced degree of dominance and present the opposite trend. Specifically, the Wuling District Ding District and the information entropy and equilibrium degree and trend of Changde City remained the same. While the Anxiang and Linli County continue to increase, other cities and counties are volatile. According to statistics, Anxiang and Linli County, primarily residential land, industrial land and land for growth of fast roads and squares; Hanson County, rapid growth is mainly residential land use, municipal facilities, public facilities, land and land slow down faster, leading to balanced 2004-2005 Entropy and degree of sag; Lixian rapid growth of housing land, industrial land and the fluctuation is mainly due to changes in green area; Taoyuan County is a balanced degree of decline of industrial land, land transportation and storage of external sites faster deceleration; Shimen living space and public facilities, growth is faster, but faster roads 
and squares of the slowdown; Tsu City, living space, roads and squares land, industrial land and public facilities land in the more volatile.

2001-2008 in the City and County of Changde, the structure of urban land use information entropy and equilibrium degree of average rankings from big to small is the Wuling District, Tsu City, Ding District, Anxiang County, Taoyuan County, Li County, Linli County, Shimen and Hanson counties. Note the past 8 years, Wu Lingqu the greatest changes in urban land use types, urban land use structure of the least orderly, balanced utilization of urban land the highest; Hanson County, and ordering the highest degree of urban land use equilibrium weakest. In 2001 the City and County of Changde City, the structure of urban land use information entropy and equilibrium degree of rank order from big to small is the Wuling District, Tsu City, Shimen, Hanshou County, Taoyuan County, Ding District, Li County, Anxiang County and Linli County, to 2008, rose to third Anxiang, while Shimen and Hanson counties were reduced to seventh and ninth. After analysis, Wu Lingqu most decentralized structure of urban land, in addition to residential land area accounts for $30 \%$, in the leading position, the rest of all kinds of urban land area of distribution was relatively better than it was in other parts of the gap; Taoyuan County and Lixian structure conversion The most obvious area of dispersion of the various types of urban land.

\section{Economic Driving Force Analysis of Changde City's urban land use structure evolution}

There are many driving force factors impacting on land use structure's evolution in actual study. The pressure of population growth is the main driving force of land use conversion in the traditional agricultural economic development times; the comparison of effectiveness of land use competition under the market mechanism is the main factor of land use change in the Industrialization stage (Zhao Cuiwei, 2006, pp.50-56). Since towns land structure mainly impacted by economic factors, this paper will analyze the economic driving force factors that impact Changde City's evolution of urban land use structure through constructing multiple regression. Multiple regression equation is as follows:

$$
\ln Y=\beta+\alpha_{1} \ln X_{1}+\alpha_{2} \ln X_{2}+\alpha_{3} \ln X_{3}+\cdots+\alpha_{n} \ln X_{n}+e
$$

In the formula, $\mathrm{Y}$ is as variables, $\beta$ is as the constant term, $\mathrm{X}_{1}, \mathrm{X}_{2}, \mathrm{X}_{3}, \ldots, \mathrm{X}_{\mathrm{n}}$ are as innumerable independent variables, $\alpha_{1}, \alpha_{2}, \alpha_{3}, \ldots, \alpha_{n}$ are innumerable independent variables corresponding to the standard coefficient, $\mathrm{e}$ is random disturbance.

Based on the availability and reliability of the material, as well as the colinearity of the selected indicators, having considered integratedly the economic driving factors that impact the urban land use structure of Changde's town land use, this paper selects the $\operatorname{GDP}\left(\mathrm{X}_{1}\right)$, the level of urbanization $\left(\mathrm{X}_{2}\right)$ and fixed assets investment $\left(\mathrm{X}_{3}\right)$ as the three economic driving force factors that impact the evolution of ChangdeCity's town land use structure. Meanwhile, it gets Changde City and county's 2001-2008 Pannel data with the corresponding index from the "Statistical Yearbook of Changde City" (2002-2009). And it sets Changde City's total area of urban land use in 2001-2008 as Y, takes the logarithm for all data, then uses E-Views software as an analytical tool to obtain the regression equation of the urban land use is:

$$
\ln Y=-0.4939+0.19202 \ln X_{1}+0.53503 \ln X_{2}+0.30465 \ln X_{3}
$$

$\mathrm{R}^{2}$ of regression model is 0.9937 , while the adjusted is 0.9935 , which illustrates the model's degree of fitting is quit good. $\mathrm{X}_{1}, \mathrm{X}_{2}, \mathrm{X}_{3}$ have all passed the examination with $1 \%$ significance level. The largest typical coefficient of the urbanization level is 0.5350 and followed by investment of fixed assets and GDP.

The level of urbanization of Changde City impacts its urban land use a lot. The level of Changde City's urbanization increased from the 8.01 in 2001 to 23.33 in 2008. The rapid growth of urbanization results in the increased demand on urban land. Therefore, the total area of the urban land use and the different types of urban land area increased correspondingly, meanwhile, the structure of urban land use changes and subsequently adjusts. For example, the urbanization level of Wu Ling District is the highest, which reached 67.14 and JInShi City's is secondly, which reached 44.16. The information entropy and equilibrium degree of their urban land use structure corresponding ranked the first and the second.

Fixed assets investment impacts the Changde City's urban land use structure secondly. The amount of fixed assets investment is its building capacity in the monetary form. The fixed assets investment of Changde City raised from 3.12605 billion yuan in 2001 to 28.47826 billion yuan in 2008, an increase of 8.11 -fold in nearly 8 years. The increased fixed assets investment results in the increased demand for the total area of urban land and the corresponding types of urban land area and the urban land use structure will also be changed and adjusted. The more investment, the greater adjustment and the larger structural changes. 
The GDP of Changde City rose from 11,15579 billion yuan in 2001 to 104,96975 billion yuan in 2008 with an average annual growth rate of $37.11 \%$. GDP's growth means the city's economic growth, and also will promote the development of the urban construction, resulting in the changes and adjustments of urban land use structure.

\section{Conclusion and discussion}

Based on the analysis on the space-time characteristics and economic driving force of the evolution of Changde City's urban land use structure in years 2001-2008, the paper finds that:

(1) the structure of urban land use information entropy is the use of integrated numerical characterization to show the urban land diversity, and is a method to reflect changes of the variety of sites in a certain period of time, which has a certain significance for land use and structural adjustment of a specific area. Nearly 8 years the structure of urban land use information entropy of Changde City first increased and then decreased slightly in nearly 8 years, while undulated slightly in 2007; equilibrium is a relatively stable situation presents. In general, more urban land use types, urban land use structure, less orderly.

(2) The decrease of entropy shows that Changde city land use system is in respect to the orderly development. The urban land structure should continue to be paid attention to adjust, intense and save in using land to maintain the orderly development of urban land. Of course, this does not means of information entropy as small as possible. As economically underdeveloped areas, Changde City should proceed from the premise of the ecology and improve the economic efficiency of urban land use to coordinate the land use structure.

(3) The level of urbanization is the main one in the economic driving force factors that affecting the evolution of urban land use structure of Changde City, followed by investment in fixed assets and GDP.

The spatial and temporal characteristics of the evolution of construction land in Changde were analyzed in this paper by using of information entropy theory. Although, to some extent, it reflects the reasonableness of land use structure of Changde City in recent years, whether the size of every land use sorts and proportion are adapted to the local economy level of development, whether it reaches the optimal configuration requirements, or whether there is a better determine standard to measure what kind of proportion of land type is best for? Then, with the overall socio-economic development, the demand for the land will have what kind of changes and what factors influence and how? This article is only considers from economic angle. but getting together with composite factors such as social development, ecological requirements and institutional, cultural, and policy environment, whose factors will impact the structure evolution of urban land, in order to more fully grasp the characteristics of urban land, make scientific and reasonable planning, which is of very essential.

\section{References}

Bo Lu, Jin Yong Zhang. (2008). Changsha City land use and driving forces of entropy. Resources and industry, 10 (6): 19-21.

Changde Municipal People's Government. Changde City Master Plan. (1999-2020), 2002, 33.

Cui Youjun. (2009). Cultivated land change in Shandong Province's economic driving forces. Modern Agricultural Sciences, 16 (8): 132-152.

Li Jiang, Qing-Sheng Guo. (2002). Based on information entropy of the dynamic evolution of urban land use structure. Yangtze River Resources and Environment, 11 (5): 393-397.

Maoliang Xiang, Lin Yanhua. (2008). Based on information entropy of the dynamic changes of land use structure. Land Resources Management, 2008, 25 (6): 48-51.

Wang Haihong, Yan-ni and so on. (2008). Structural Evolution of land for construction in Gansu Province and its driving forces. Arid Land Resources and Environment, 22 (8): 1-6.

Wang Qun, Wan-mao. (2005). Chinese provinces between drivers of land use empirical study. China Land Science, 2005, 19 (6): 21-25.

Wan-mao, Han Tongkui. (2002). Land use planning. Beijing: China Agriculture Press.

Wetland restoration, Wu-fang. (2003). Regional land use structure of the law of information entropy. Journal of Natural Resources, 18 (1) :112-117.

Zhang study, Yao Hui, etc. (2006). Based on information entropy in Jinan urban and rural land use structure and dynamics of the distribution. Land Resources Management, 23 (2): 74-78.

Zhao Cuiwei. (2006). Study land use and so on. Different stages of economic development Comparative study of land use change - in Guangxi and Jiangsu Wujiang Jiangzhou example. Resources Science, 2006, 28 (5): 50-56. 
Table 1. Information entropy, equilibrium degree and advantage degree of urban land structure in Changde from 2001-2008

\begin{tabular}{|c|c|c|c|c|c|c|c|c|}
\hline & 2001 & 2002 & 2003 & 2004 & 2005 & 2006 & 2007 & 2008 \\
\hline $\begin{array}{c}\text { Residential Land } \\
(\%)\end{array}$ & 0.3481 & 0.3351 & 0.3226 & 0.3221 & 0.3165 & 0.2886 & 0.2821 & 0.2987 \\
\hline Public Facilities (\%) & 0.1794 & 0.1754 & 0.1798 & 0.1767 & 0.1771 & 0.1947 & 0.1828 & 0.1839 \\
\hline Industrial land (\%) & 0.1653 & 0.1827 & 0.1759 & 0.1763 & 0.1766 & 0.2181 & 0.2372 & 0.2231 \\
\hline Storage Space (\%) & 0.0433 & 0.0421 & 0.0422 & 0.0415 & 0.0406 & 0.0359 & 0.0338 & 0.0315 \\
\hline $\begin{array}{c}\text { External transport } \\
\text { site (\%) }\end{array}$ & 0.0605 & 0.0591 & 0.0595 & 0.0626 & 0.0656 & 0.0580 & 0.0537 & 0.0513 \\
\hline Road Plaza site (\%) & 0.1169 & 0.1183 & 0.1272 & 0.1268 & 0.1246 & 0.1197 & 0.1118 & 0.1164 \\
\hline $\begin{array}{c}\text { Sites of municipal } \\
\text { facilities (\%) }\end{array}$ & 0.0468 & 0.0497 & 0.0511 & 0.0496 & 0.0524 & 0.0472 & 0.0445 & 0.0437 \\
\hline Green (\%) & 0.0397 & 0.0377 & 0.0417 & 0.0444 & 0.0468 & 0.0379 & 0.0541 & 0.0513 \\
\hline EntropyH & $\mathbf{1 . 8 0 1 0}$ & $\mathbf{1 . 8 0 8 0}$ & $\mathbf{1 . 8 2 7 4}$ & $\mathbf{1 . 8 3 1 8}$ & $\mathbf{1 . 8 4 2 7}$ & $\mathbf{1 . 8 1 6 1}$ & $\mathbf{1 . 8 2 1 7}$ & $\mathbf{1 . 8 0 8 1}$ \\
\hline $\begin{array}{c}\text { Equilibrium } \\
\text { degreeJ }\end{array}$ & $\mathbf{0 . 8 6 6 1}$ & $\mathbf{0 . 8 6 9 4}$ & $\mathbf{0 . 8 7 8 8}$ & $\mathbf{0 . 8 8 0 9}$ & $\mathbf{0 . 8 8 6 2}$ & $\mathbf{0 . 8 7 3 3}$ & $\mathbf{0 . 8 7 6 0}$ & $\mathbf{0 . 8 6 9 5}$ \\
\hline DominanceI & $\mathbf{0 . 1 3 3 9}$ & $\mathbf{0 . 1 3 0 6}$ & $\mathbf{0 . 1 2 1 2}$ & $\mathbf{0 . 1 1 9 1}$ & $\mathbf{0 . 1 1 3 8}$ & $\mathbf{0 . 1 2 6 7}$ & $\mathbf{0 . 1 2 4 0}$ & $\mathbf{0 . 1 3 0 5}$ \\
\hline
\end{tabular}

Table 2. The urban land structure of information entropy in Changde city and towns from 2001-2008

\begin{tabular}{|c|c|c|c|c|c|c|c|c|}
\hline & 2001 & 2002 & 2003 & 2004 & 2005 & 2006 & 2007 & 2008 \\
\hline Changde & 1.8010 & 1.8080 & 1.8274 & 1.7485 & 1.8427 & 1.8161 & 1.8217 & 1.8081 \\
\hline Wuling & 1.8162 & 1.8254 & 1.8501 & 1.8493 & 1.8668 & 1.8339 & 1.8361 & 1.8223 \\
\hline Dincheng & 1.6462 & 1.6562 & 1.6497 & 1.6685 & 1.6603 & 1.6518 & 1.6507 & 1.6441 \\
\hline Anxiang & 1.5914 & 1.6027 & 1.6083 & 1.6761 & 1.6643 & 1.6705 & 1.7026 & 1.7088 \\
\hline Hanshou & 1.7383 & 1.6365 & 1.7367 & 1.7213 & 1.3767 & 1.3862 & 1.3751 & 1.3751 \\
\hline Li & 1.6323 & 1.6439 & 1.6567 & 1.5624 & 1.6242 & 1.6218 & 1.6737 & 1.6794 \\
\hline Linli & 1.5446 & 1.6086 & 1.6230 & 1.6262 & 1.6262 & 1.6556 & 1.6532 & 1.6553 \\
\hline Taoyuan & 1.6573 & 1.6865 & 1.7211 & 1.7184 & 1.7184 & 1.5526 & 1.5211 & 1.5338 \\
\hline Shimen & 1.7527 & 1.8091 & 1.8525 & 1.4599 & 1.4599 & 1.5089 & 1.5085 & 1.5554 \\
\hline Jinshi & 1.7817 & 1.8480 & 1.7425 & 1.7245 & 1.7163 & 1.8628 & 1.8628 & 1.8628 \\
\hline
\end{tabular}

Table 3. The urban land structure of equilibrium degree in Changde city and towns from 2001-2008

\begin{tabular}{|c|c|c|c|c|c|c|c|c|}
\hline & 2001 & 2002 & 2003 & 2004 & 2005 & 2006 & 2007 & 2008 \\
\hline Changde & 0.8661 & 0.8694 & 0.8788 & 0.8809 & 0.8862 & 0.8733 & 0.8760 & 0.8695 \\
\hline Wuling & 0.8734 & 0.8778 & 0.8897 & 0.8893 & 0.8977 & 0.8819 & 0.8830 & 0.8763 \\
\hline Dincheng & 0.7917 & 0.7965 & 0.7933 & 0.8024 & 0.7984 & 0.7943 & 0.7938 & 0.7906 \\
\hline Anxiang & 0.7653 & 0.7707 & 0.7735 & 0.8061 & 0.8004 & 0.8033 & 0.8188 & 0.8218 \\
\hline Hanshou & 0.8360 & 0.7870 & 0.8352 & 0.8278 & 0.6621 & 0.6666 & 0.6613 & 0.6613 \\
\hline Li & 0.7850 & 0.7905 & 0.7967 & 0.7514 & 0.7811 & 0.7799 & 0.8049 & 0.8076 \\
\hline Linli & 0.7428 & 0.7736 & 0.7805 & 0.7820 & 0.7820 & 0.7962 & 0.7950 & 0.7960 \\
\hline Taoyuan & 0.7970 & 0.8110 & 0.8277 & 0.8264 & 0.8264 & 0.7466 & 0.7315 & 0.7376 \\
\hline Shimen & 0.8429 & 0.8700 & 0.8909 & 0.7020 & 0.7020 & 0.7256 & 0.7254 & 0.7480 \\
\hline Jinshi & 0.8568 & 0.8887 & 0.8380 & 0.8293 & 0.8254 & 0.8958 & 0.8958 & 0.8958 \\
\hline
\end{tabular}




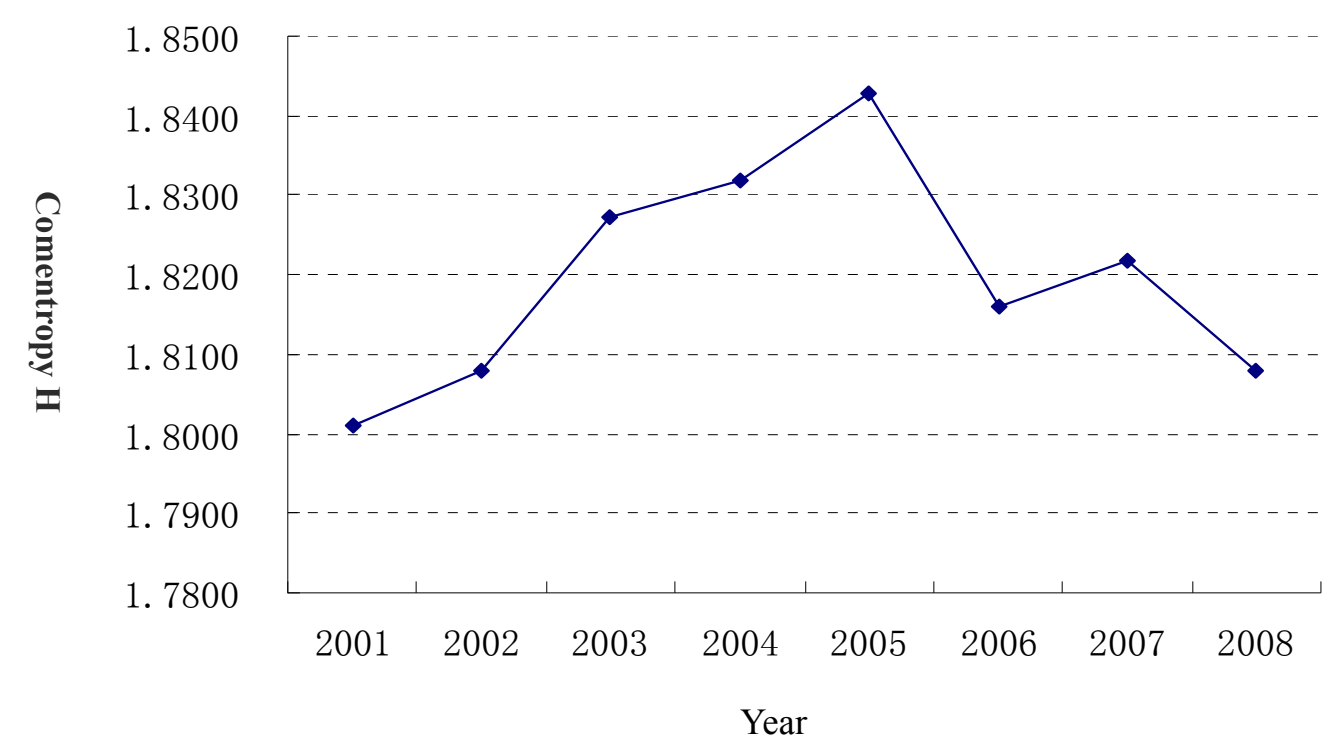

Figure 1. Urban land structure change of information entropy in Changde from 2001-2008 